\title{
Evolución histórica y ambiental en los procesos de trasformación del lago Chapala*
}

\section{Historical and Environmental Evolution in the Transformation Processes of Lake Chapala}

\section{Carmen Pedroza-Gutiérrez ${ }^{* *}$, Juan Manuel Catalán-Romero***}

Recibido: 2016-05-30 // Aprobado: 2016-08-30 // Disponible en línea: 2017-01-30

Cómo citar este artículo: Pedroza-Gutiérrez C. y Catalán Romero, J. M. (2017). Evolución histórica y ambiental en los procesos de trasformación del lago Chapala. Ambiente y Desarrollo, 21(40), 09-25. https://doi.org/10.11144/Javeriana.ayd21-40.ehap

doi:10.11144/Javeriana.ayd21-40.ehap

\section{Resumen}

El lago de Chapala ha sufrido grandes transformaciones que han afectado su capacidad productiva. Esta situación se ve reflejada en los cambios del territorio que conforma su ribera, ya que los usos y el control del territorio ribereño han afectado negativamente sus perspectivas y potencial productivo. Este trabajo tiene como objetivo hacer un recuento histórico de factores que han detonado las transformaciones del territorio ribereño del lago de Chapala y, a su vez, cómo estas transformaciones han afectado su capacidad productiva. Los resultados indican que, históricamente, el lago ha sido sometido a un uso excesivo de sus recursos determinado por los modelos de desarrollo que ha seguido el país y de acuerdo con una mala comprensión de los ecosistemas lacustres.

Palabras clave: cambios de uso del suelo; lago de Chapala; ecosistemas lacustres; pesca

\footnotetext{
Este proyecto fue financiado por el proyecto de investigación PAPIIT IA300215 de la Universidad Nacional Autónoma de México (UNAM) y el proyecto de investigación SIP-20150431 de la Secretaría de Investigación y Posgrado del Instituto Politécnico Nacional (IPN).

** Doctora en Socioeconomía del Desarrollo, investigadora de tiempo completo en la Unidad Académica de Estudios Regionales (UAER) de la Universidad Nacional Autónoma de México. Correo electrónico: pedrozacarmen@yahoo.com. Autora de correspondencia.

*** Doctor en Pedagogía, profesor-investigador del Centro Interdisciplinario de Investigación para el Desarrollo Integral Regional Instituto Politécnico Nacional. Correo electrónico: jmcatalan@ipn.mx
} 


\begin{abstract}
Lake Chapala has undergone important transformations that affected its productive capacity. This situation is reflected in the changes of its coastline territory, given that the uses and control of the coastal territory have negatively affected its prospects and productive potential. This work aims to make a historical account of the factors that have triggered the transformations of the coastal territory of Lake Chapala and, at the same time, how these transformations have affected its productive capacity. Results indicate that, historically speaking, the lake has been subject to excessive use of its resources as determined by the development models followed by the country and based on a poor understanding of lake ecosystems.
\end{abstract}

Keywords: land use changes; Lake Chapala; lake ecosystems; fishing 


\section{Introducción}

Los cuerpos de agua continentales son los ecosistemas que han sufrido los cambios más radicales en los últimos cien años como resultado de actividades antropogénicas (Welcomme et al., 2010). Las cuencas hidrográficas son transformadas y deterioradas en razón de las diferentes actividades económicas que se llevan a cabo en ellas o a partir de ellas. Algunos de los impactos evidentes que han sufrido son los cambios en su morfología y en el territorio costero o en su perfil ribereño.

Los lagos someros tropicales han sido tomados como ejemplo de transformaciones radicales de morfología lacustre, de cambio de uso del suelo y del control de su indefinido territorio costero, ya que debido a las oscilaciones naturales e inducidas del nivel de sus aguas esta superficie es comúnmente variable (Coops et al., 2003; Kolding y van Zwieten, 2012), característica que incentiva cambios en su uso.

Las costas del lago de Chapala, un lago tropical somero en el occidente mexicano, han sido objeto de grandes transformaciones causadas por diferentes formas de apropiación de los recursos naturales de la región de la que forma parte, además de los cambios propios de su naturaleza fisiográfica. Dichas transformaciones se han dado de forma natural o inducida por la mano del hombre y están relacionadas con dos factores: la belleza paisajística del lago y las diferentes perspectivas que el país ha seguido en sus modelos de desarrollo.

Los diferentes modelos productivos que han tenido cabida en la región chapálica, empezando por las estancias ganaderas y continuando con la hacienda y el ejido, han sido una respuesta a las demandas de los mercados nacionales e internacionales (Dávila, 2014). Dichos modelos productivos se han dado bajo la influencia o demanda de modelos externos de desarrollo que, por lo general, han tenido como base un uso desmedido de los recursos naturales, tanto terrestres como acuáticos. Asimismo, han privilegiado el desarrollo de actividades terrestres como la ganadería o la agricultura, dejando de lado la pesca, que ha ido perdiendo cada vez más los espacios costeros y los recursos acuáticos del lago que pudiesen incentivar su desarrollo.

Desde hace décadas la agricultura parece ser la actividad dominante en los planes de desarrollo de varios países del mundo y, a partir de la motivación para desarrollarla, se diseñan e implementan políticas que no siempre consideran la existencia de otros recursos o la necesidad de conservar los recursos hídricos. Sin embargo, además del intenso desarrollo agrícola que sostiene el lago de Chapala, también se ha desarrollado una amplia industria manufacturera que depende de su cuenca y una importante industria turística que ha aprovechado su belleza paisajística.

De acuerdo con las anteriores consideraciones, este trabajo tiene como objetivo identificar los detonantes de las transformaciones del territorio ribereño del lago de Chapala y, asimismo, cómo estas han afectado su capacidad productiva.

Para poder abordar nuestro objetivo, en primer lugar definimos las particularidades de los lagos someros tropicales como es el caso del lago de Chapala. Describimos también su cuenca, incluyendo la actividad pesquera de corte artesanal. Posteriormente, realizamos un análisis histórico-político del manejo y la apropiación de los recursos hídrico-terrestres, a nivel de cuenca y en el vaso lacustre, identificando acciones y procesos de diversos actores, y concluimos con algunas consideraciones finales.

\section{Metodología. Zona de estudio y localización}

\section{La cuenca Lerma-Chapala}

La cuenca Lerma-Chapala tiene una extensión de $53591 \mathrm{~km}^{2}$, parte de los cuales se encuentran distribuidos en los estados de Guanajuato (43.8\%), Michoacán (30.3\%), Jalisco (13.4\%), México 
(9.8\%) y Querétaro (2.8\%). Esto incluye 205 municipios. La población de la cuenca, considerando los municipios que tienen algún territorio dentro de ella, se calcula en aproximadamente 15180376 habitantes, lo cual representa un $15.6 \%$ del total nacional. Sin embargo, el área de influencia de la cuenca contaría con 36537144 habitantes (38\% del total nacional). Esta zona a su vez representa el $47 \%$ del valor agregado censal bruto (VACB) nacional (Cardona, 2004). A este valor, el mayor porcentaje lo aporta la industria manufacturera $(38.76 \%)$ y el menor la actividad pesquera $(0.01 \%)$.

\section{El lago de Chapala}

El lago de Chapala es parte del sistema hídrico de la cuenca y está clasificado como un lago tropical poco profundo. Su superficie actual cubre aproximadamente 75 por $22 \mathrm{~km}$ en promedio y su profundidad oscila entre 6 y $11 \mathrm{~m}$ (Tereshchenko et al., 2002). Este lago es el más grande en México y el tercero más grande en América Latina, después del lago Titicaca en los Andes y el lago Nicaragua en Centroamérica. Sin embargo, en cuanto que lago tropical poco profundo, hay autores que afirman que el de Chapala se puede considerar el más grande del mundo (Sandoval, 1994). Sus ríos tributarios más importantes son el Duero y el Lerma. El lago es compartido por dos estados: Jalisco, donde se ubica el $86 \%$ de su superficie, y Michoacán, que posee el $14 \%$. Su ribera está dividida en diez municipios: Chapala, Jamay, Jocotepec, La Barca, Ocotlán, Tizapán el Alto y Tuxcueca, todos estos en Jalisco, y Cojumatlán y Venustiano Carranza en Michoacán (figura 1).

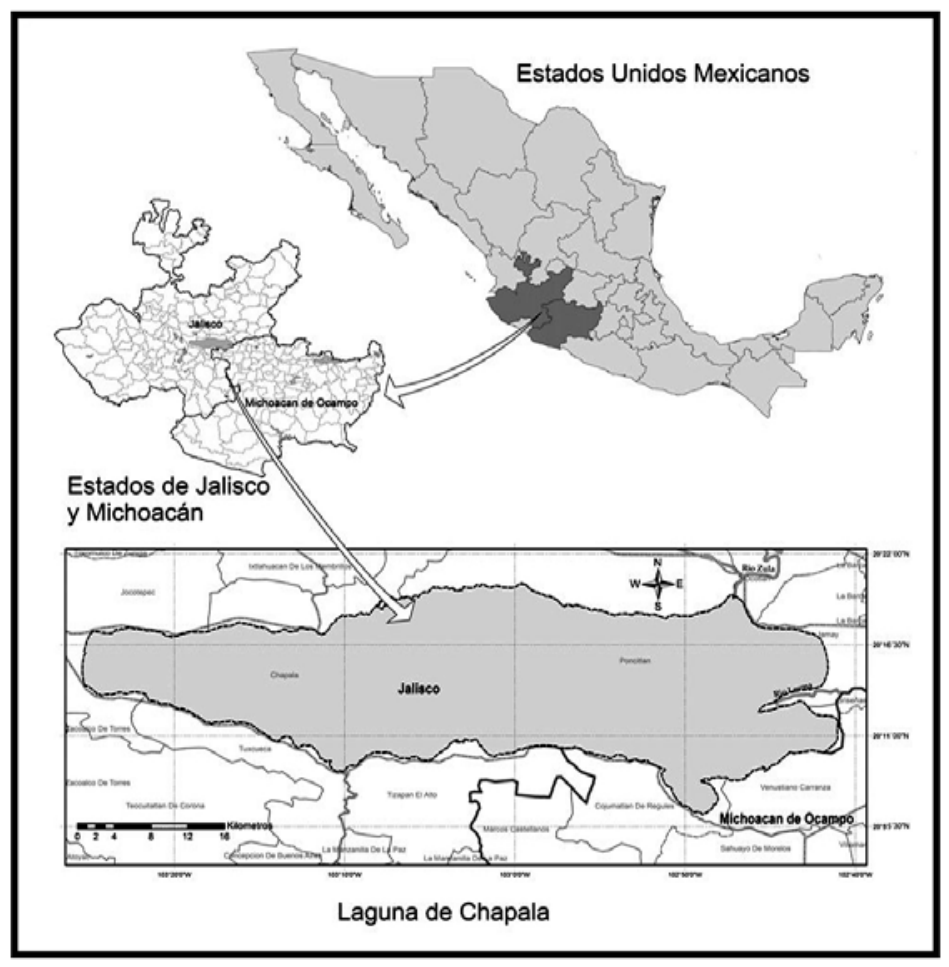

Figura 1. Localización del lago de Chapala

Fuente: INEGI (2010). Edición: Fabián Villalpando Barragán 
En la actualidad el lago de Chapala cuenta con aproximadamente 2401 pescadores, conjuntados en 67 asociaciones de diversos tipos. La Carta Nacional Pesquera 2006 registra 37000 artes de pesca y 1219 embarcaciones. Las principales pesquerías son la tilapia (54\%) (Oreochromis spp.), la carpa (34\%) (Carassius spp.) y el charal (11\%) (Chirostoma spp.). La carpa y la tilapia son especies introducidas, mientras que el charal, junto con el pescado blanco (Chriostoma lucius) y el bagre (Ictalurus ochoterenai) son parte del grupo de especies nativas que constituían la biodiversidad del lago y que hoy son significativamente escasas, al igual que el pescado blanco.

\section{Trabajo de campo}

En primera instancia se llevó a cabo una revisión historiográfica y se consultó el archivo para recopilar información sobre las características y las transformaciones del lago de Chapala y su ribera. Además, se consultó el archivo histórico de la ciudad de Chapala, donde se recopilaron fotografías históricas que muestran los cambios en algunas partes del litoral del lago y de cómo se llevaba a cabo la actividad pesquera a finales del siglo XIX.

Posteriormente, se llevó a cabo un recorrido por la ribera del lago para observar cómo se reflejan en la actualidad sus transformaciones a través de la construcción de infraestructura y el desarrollo de diferentes actividades económicas. Esto se documentó tomando fotografías de sus puertos principales: Chapala, Jocotepec y Jamay, en Jalisco, y La Palma y Petatán en Michoacán. Asimismo, se consultaron bases de datos estadísticas sobre los niveles de agua del lago y su productividad pesquera.

\section{Detonantes naturales e inducidos del cambio}

Para poder entender la diversidad de factores que han intervenido en las transformaciones, los usos y el control del territorio costero del lago de Chapala es necesario conocer su génesis natural y antrópica. Existen factores naturales inherentes al lago que se derivan de sus características de lago tropical somero y, además, factores inducidos por la mano del hombre como consecuencia de la puesta en obra de diversos enfoques productivos que ha seguido el país a lo largo de su historia.

\section{Los lagos someros tropicales}

Los lagos tropicales poco profundos se caracterizan por tener amplias fluctuaciones en su nivel de agua (Sarch y Birkett, 2000; Coops et al., 2003; Kolding y van Zwieten, 2012). Estos lagos y los embalses hechos por el hombre son los que en general experimentan los cambios más acentuados y también los volúmenes de producción pesquera más altos por unidad de área, debido a que las fluctuaciones en el nivel de agua generan una mezcla resultante del flujo de sus afluentes cuyo reciclaje es la causa de la generación de nutrientes que son, a su vez, el origen de una mayor productividad (Kolding y van Zwieten, 2012).

La amplitud de las fluctuaciones en el nivel del agua de los lagos depende tanto de las características climáticas de cada región como de los usos humanos (Coops et al., 2003). Se ha demostrado que ambos factores pueden alterar de manera radical el estado de los lagos someros tropicales y por lo tanto la actividad pesquera que se practica en ellos (Sarch y Birkett, 2000; Dudgeon, 2000; Moncayo et al., 2012). Otros fenómenos que influyen en las oscilaciones en los niveles de agua en estos lagos son la evaporación y la filtración, además del nivel de agua que entra por sus afluentes y la lluvia (Sarch y Birkett, 2000).

La estacionalidad y los volúmenes de las descargas de agua tienen influencia en la flora y en la fauna de los ríos, en particular en los peces, pues provocan alteraciones en los periodos de escasez (durante la época de secas) y en los periodos de abundancia de recursos (durante la época de lluvias) (Dudgeon, 2000). Se ha comprobado que el descenso en los volúmenes de producción pesquera está sin duda relacionado con las oscilaciones en los niveles de agua de los lagos. 


\section{Usos humanos}

Por otra parte, diferentes actividades socioeconómicas dependen del agua proporcionada por lagos y ríos que forman parte de cuencas hidrográficas. Muchas veces, para llevar a cabo estas actividades se efectúan transformaciones de estos sistemas naturales, las cuales a su vez pueden resultar en diferentes tipos de deterioro ambiental. El impacto de los factores de cambio inducidos por la mano del hombre sobre los recursos hídricos ha sido reconocido a escala mundial y uno de ellos es el daño a la biodiversidad asociada a estos sistemas hídricos.

Las amenazas de origen antrópico tienen su origen en el rápido crecimiento del sector industrial, los desarrollos turísticos y los complejos urbanos, cuyas demandas de agua potable se traducen en su contaminación y en la sobreexplotación de los mantos acuíferos (Dudgeon, 2000; von Bertrab, 2003). Aunado a esto, en épocas de estiaje los litorales de los lagos someros tropicales suelen ser utilizadas para la práctica de actividades agropecuarias (Sarch y Birkett, 2000).

Según Dudgeon (2000), en un estudio llevado a cabo en Asia sobre las amenazas de las actividades humanas en cuencas hídricas se sugiere que estas se pueden clasificar en cuatro categorías: alteraciones del flujo de agua (esto puede ser a través de la construcción de presas o la extracción de agua para riego); contaminación y alteración en el drenado (azolve causado por la deforestación) y sobreexplotación (sobrepesca). Dichas amenazas coinciden con lo sugerido por Beeton (2002), quien además de estas identifica la eutrofización, la introducción de especies exóticas y los cambios de uso del suelo.

Además de lo anterior, las zonas costeras de ríos y lagos son espacios considerados altamente atractivos para realizar actividades de recreación y turismo. El turismo desarrollado en estas áreas tiene características, potenciales y amenazas similares en todos los cuerpos de agua donde se lleva a cabo. Algunos de los problemas son desarrollos turísticos mal planeados y con un gran impacto ambiental, además de que la infraestructura afecta la estética y belleza del paisaje natural (Gattenlöhner, 2006).

\section{Transformaciones y apropiación del litoral del lago de Chapala. Un pro- blema multifactorial histórico-moderno}

Las transformaciones y formas de apropiación de los recursos de la zona costera del lago de Chapala tienen que ver con la transformación general de su cuenca - la Lerma-Chapala- y con los cambios llevados a cabo directamente en la ribera del lago.

Según Cotler (2004), son dos las razones que explican la situación de deterioro de la cuenca Lerma-Chapala: 1) el modelo de desarrollo hidráulico empleado para satisfacer las demandas de los sectores productivos y de las ciudades, y 2) el hecho de que el Gobierno quiera resolver un problema ecosistémico manipulando solo el recurso agua.

Ambos elementos tienen su origen en la relación entre las formas de explotación y apropiación de los recursos naturales, las dinámicas de los mercados mundiales y las medidas tomadas para adaptarse a estos.

En México desde la época colonial se fue gestando un nuevo paradigma en cuanto a las formas de manejo y apropiación del territorio. Desde aquella época se ha tenido la visión de que la explotación a gran escala de la riqueza natural del país es la clave para el crecimiento económico (Simonian, 1995). Así se da inicio al cambio hacia nuevas formas de explotación de los recursos naturales y el surgimiento de diferentes modelos de desarrollo.

En el modelo colonial, entre los siglos XVI y XIX, predomina el desarrollo de la agricultura y la ganadería. En las últimas décadas del siglo XVI se crean las estancias ganaderas, las cuales fueron el resultado de la organización de la propiedad, el trabajo y la producción. Posteriormente, a mediados del siglo XVIII, al empezar a fraccionarse se convertirían en haciendas (Dávila, 2014). El interés por la ganadería 
se da por ser la actividad que más rápidamente generaba utilidades y no requería mucha mano de obra. Por otra parte, el incremento poblacional ocurrido de la Conquista hasta la primera mitad del siglo XVIII (García de León, 1988) es otro incentivo para el aumento de la ganadería, y conforme aumenta la necesidad por la carne y los productos derivados del ganado se va incrementando la frontera ganadera. Esto trae como consecuencia la transformación del paisaje natural y los cambios de uso del suelo, de agrícola y lacustre a ganadero, ya que algunas zonas se drenaron y desecaron (Covarrubias y Ojeda, 2009). Así, a medida que la agricultura va adquiriendo importancia, la pesca va perdiendo terreno.

Sin embargo, no es hasta el gobierno de Porfirio Díaz (1884-1911), en los albores del siglo XX, que la hacienda se convierte en la base del modelo de desarrollo liberal, de la producción agropecuaria y de la organización socioeconómica del país. La hacienda es una prueba más del intento de adopción del modelo europeo de expansión e industrialización, ya que para Díaz este era el símbolo del progreso y la modernidad (Dávila, 2014).

Estos enfoques de modernización y desarrollo han tenido impactos negativos graves en los sistemas lacustres del país. El modelo productivista de las haciendas refleja una apropiación del territorio que afecta el litoral costero lacustre y por ende a la actividad pesquera, ya que durante esta época el uso y control del territorio se encontraba en las manos de los hacendados que tenían el poder para efectuar grandes transformaciones en los ecosistemas lacustres. Algunos ejemplos de ello fueron la desecación de la laguna de Mayrán y la de Viesca en el estado de Coahuila (Wolfe, 2013). En Michoacán podemos citar el caso de la ciénaga de la laguna de Zacapu hacia 1900 y poco después la de Chapala (19041908). Otro ejemplo más reciente de la continuidad de este modelo, que promueve la extensión de la frontera agrícola a través de la desecación de cuerpos de agua superficiales, es la laguna de Colesio, en el valle de Ecuandureo, también en Michoacán, llevada a cabo por la entonces Secretaría de Recursos Hidráulicos (SRH) en 1967 (González Santana, 2009).

La Revolución y la consecuente reforma agraria apoyada por la creación de la Comisión Nacional de Irrigación en 1926 incentivan la agricultura e incrementan el uso del agua. Continúa la evolución socioeconómica del país con la tendencia predominante entre los agentes gubernamentales a considerar sinónimo de desarrollo el crecimiento económico basado en la industrialización. Simultáneamente, se inicia una lenta pero constante recuperación demográfica, así como una expansión de los centros urbanos.

\section{Las transformaciones en la cuenca Lerma-Chapala}

El caso de la cuenca Lerma-Chapala en general y del lago de Chapala en particular, es un ejemplo fehaciente de cómo los paisajes acuáticos y terrestres se modifican debido a las demandas de un modelo de desarrollo que impone un crecimiento económico con base en la utilización desmedida y mal planificada de los ecosistemas que conforman una región.

A través de la historia de México esta cuenca ha sido manejada teniendo en cuenta los distintos enfoques de desarrollo adoptados por el Estado mexicano y que han sido la causa principal de la transformación y el deterioro de sus recursos naturales. Una prueba de ello es que entre 1970 y el 2006 se redujo el $30 \%$ de su cobertura vegetal y el $21 \%$ de sus cuerpos de agua (Cotler, 2004). Además, el extenso crecimiento industrial desarrollado a partir del uso y la apropiación de sus recursos ha tenido como consecuencia la contaminación del agua.

Hoy en día a lo largo de la cuenca existen cerca de 3500 industrias que realizan actividades dentro de los ramos metal-mecánico, de curtido de pieles, electromecánico, químico, pinturas, de baterías electrolíticas y petroquímica. Estas industrias vierten parte de sus desechos en los cuerpos de agua que conforman la cuenca. Así, en sus diferentes niveles alto, bajo y medio, la cuenca presenta grados de contaminación que varían entre contaminado y altamente contaminado, por lo cual el agua que llega al lago de Chapala ya no es de buena calidad. Ojeda (2007) reporta que en el 2001 
el $64 \%$ del agua que llegaba al lago no había sido tratada, pues las plantas tratadoras de agua que existen a lo largo de la cuenca son insuficientes, además de que la mayoría no operan, o lo hacen en solo 50 o $70 \%$ de su capacidad.

En la actualidad, además de las industrias a lo largo de la cuenca, existen 552 presas que alteran y disminuyen el flujo de agua del río Lerma al lago de Chapala (Cotler y Gutiérrez, 2005). Estas forman parte del sistema de irrigación mexicano, que es uno de los más amplios del mundo debido en parte a que el $76.7 \%$ del agua dulce superficial está destinada para uso agrícola (Comisión Nacional del Agua [Conagua], 2011).

Además de las presas y los bordos registrados están los que no son autorizados ni regulados, los cuales han sido construidos al amparo de la falta de vigilancia y a partir de la necesidad que tiene el sector agrícola del preciado líquido. Asimismo, la actividad agrícola, tanto de riego como de temporal, ha llegado a realizarse gracias a concesiones otorgadas temporalmente por Conagua, concesiones que han derivado en conflictos sociales.

Estos fenómenos se dieron conjuntamente con un gran crecimiento demográfico que es consecuencia directa de la expansión del mercado de trabajo. El crecimiento poblacional originó mayor urbanización e incremento en la demanda de agua, acompañado del aumento del volumen de aguas residuales urbanas y de las procedentes tanto de las industrias como de la porcicultura.

De acuerdo con lo expuesto en los acápites precedentes, los principales problemas de la cuenca son: sobreexplotación, contaminación y cambios en los flujos de agua ocasionados por las presas y la infraestructura hídrica que se ha construido en la región sin tener en cuenta los costos ecológicos que conlleva. Y el río Lerma es el principal depositario de los residuos de las industrias, de la porcicultura y de las poblaciones humanas (Ojeda y Covarrubias, 2008) que vierten las aguas residuales y sin tratamiento en el lago, con lo cual se afecta su calidad y disminuyen sus niveles.

\section{Transformaciones en el lago de Chapala}

Los factores que han transformado la ribera del lago de Chapala se pueden agrupar en tres: 1) los naturales, derivados de las características fisiológicas del lago; 2) los antropogénicos, como la expansión de la agricultura y la ganadería, y 3) el desarrollo de infraestructura para el turismo y zonas urbanas. Todos estos elementos han tenido impactos negativos sobre la actividad pesquera, ya sea afectando los niveles y calidad de agua del lago, o ya sea apropiándose de espacios donde originalmente se llevaba a cabo la pesca.

Los cambios naturales del lago se deben principalmente a su naturaleza como lago tropical somero, lo cual se refleja en las fluctuaciones en su nivel de agua (véase figura 11). Otros fenómenos naturales que han afectado los niveles de agua del lago son los cambios en los niveles de precipitación pluvial, niveles que han experimentado drásticas variaciones que van de los 500 a los $1000 \mathrm{~mm}$ de agua al año y que han sido influidas por fenómenos naturales como El Niño (Tereshchenko et al., 2002). La evaporación, por su parte, puede llegar a sumar $10 \mathrm{~mm}$ diarios, principalmente en los meses más calurosos de abril y mayo (Filonov et al., 1998; Tereshchenko et al., 2002).

Las transformaciones de origen antrópico datan de la época de la Colonia cuando en la ciénaga de Chapala comienza un proceso de acaparamiento de la tierra que se intensifica en el siglo XVIII. Primero, con la creación de estancias ganaderas y posteriormente con el surgimiento de la hacienda de Guaracha, a finales del siglo XVII y principios del XVIII. La hacienda se convierte en brazo ejecutor de un modelo de desarrollo que favorecía la agricultura y la ganadería. Desde entonces, los suelos que se iban descubriendo del lago de Chapala en época de estiaje se convirtieron en zonas atractivas para el desarrollo de la ganadería, y después para la agricultura debido a su alta fertilidad. 
Desde finales del siglo XVIII y durante el XIX los dueños de la hacienda de Guaracha acrecentaron su patrimonio a través del despojo de tierras de comunidades indígenas, por lo cual en la segunda mitad del siglo XIX la hacienda era considerada un gran latifundio de baja productividad agrícola y cuya principal actividad era la ganadería extensiva (Dávila, 2014). Sin embargo, durante el último tercio del siglo XIX la hacienda se transforma y adquiere maquinaria moderna, lo que la convierte en un centro agroindustrial preponderante y con impactos decisivos para la región. De esta manera, a finales del siglo XIX controlaba más de 100000 ha dedicadas a la actividad agrícola y ganadera.

La pesca en el lago de Chapala, por su parte, es una actividad que se ha llevado a cabo desde épocas prehispánicas, y además de proveer alimento a los pueblos lacustres los excedentes siempre han sido objeto de comercialización y una forma de obtención de ingresos para los pescadores y quienes participan en su transformación y distribución. Sin embargo, el papel de la pesca ha pasado por diferentes etapas, durante las cuales no siempre se ha incentivado su potencial, sino más bien se ha descuidado por darle preferencia a actividades agropecuarias y turísticas.

En el periodo colonial la pesca en el lago continuó, aunque como una actividad complementaria pero importante dada la variedad y la abundancia de peces (Clavijero, 1964). Esta abundancia aunada a la escasez y el despojo de tierras provocó que la pesca recobrara importancia, y así surgieron poblados de pescadores quienes además de pescar para autoconsumo proveían de pescado a ranchos y haciendas ganaderas, además de que el sabor y calidad del pescado del lago eran ya bien conocidos, razón por la cual este ya llegaba a ciudades como Guadalajara (Ortiz, 2001).

La abundancia de pescado y la falta de tierras hacen de la pesca una actividad económica importante desde la Colonia hasta finales del siglo XIX. De hecho, en aquella época se empleaban en la pesca pueblos completos, ya que toda la familia participaba, algunos en la captura y otros en el proceso y la comercialización. Ortiz (2001) asegura que los volúmenes de captura eran tan abundantes que los acaparadores de pescado tenían trabajando en las orillas del lago a un ejército de hombres y mujeres que preparaban el pescado para su comercialización. Las figuras 2 y 3 , que son fotografías hechas a finales del siglo XIX y principios del XX, muestran cómo la ribera del lago estaba dedicada a la actividad pesquera y no competía por espacios con otras actividades económicas.

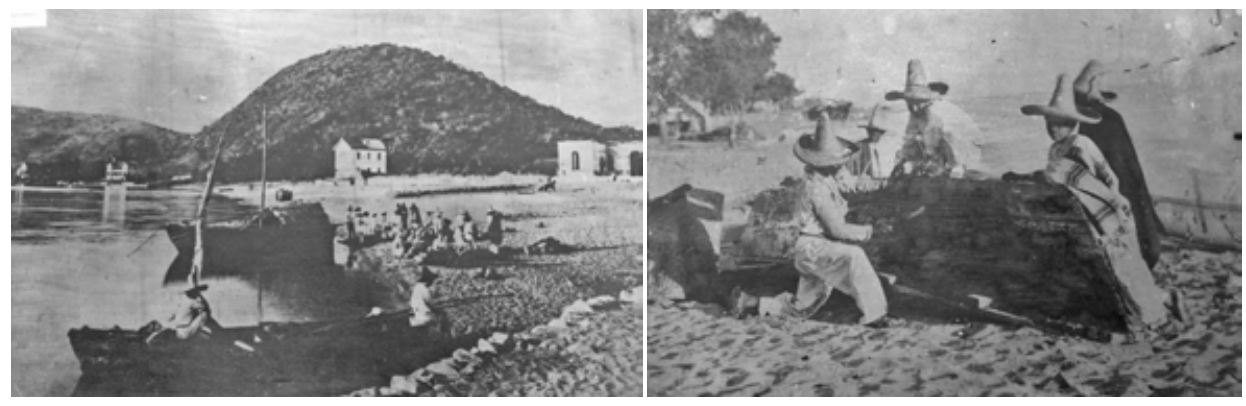

Figuras 2 y 3. Ribera del lago de Chapala a finales del siglo XIX

Fuente: Archivo histórico del municipio de Chapala 
Posteriormente, el deseo de expansión de los hacendados incentiva a que entre 1904 y 1909 Porfirio Díaz permita la desecación inducida más grande en la historia del lago. Con el pretexto de que la zona de humedales era poco profunda y se encontraba seca en parte del año, se procede a desecar un aproximado de 50000 ha, a través de la construcción de un bordo. Estas nuevas tierras se dedicarían a la actividad agrícola, pues en ese momento histórico la agricultura y la ganadería se visualizaban como las mejores opciones de desarrollo del país, mientras que la pesca en aguas continentales se consideraba una actividad poco rentable.

Además de lo anterior, a finales del siglo XIX y principios del XX la pesca se ve afectada por las leyes porfiristas. Por una parte, se impone una nueva ley de acuerdo con la cual se expropian las corrientes y depósitos de agua para convertirlos en propiedad federal, y por otra, se prohíbe la pesca a todas aquellas personas que no contaran con el respectivo permiso.

La desecación benefició a los hacendados ya que las zonas de cultivo se ampliaron y se intensificó la producción agrícola. Sin embargo, se truncó la función natural de los ríos Lerma y Duero que durante los meses de junio y octubre cubrían de agua y fertilizaban la región, mientras que entre noviembre y mayo quedaban descubiertos los terrenos lacustres (Moreno, 1989). Para el lago de Chapala este proceso también tuvo impactos negativos, puesto que afectó sus ciclos naturales y disminuyó su capacidad de adaptarse y autorregularse (Dávila, 2014).

En 1936 el reparto agrario y el establecimiento del ejido en la región cambió profundamente el uso y las formas de apropiación del territorio, así como la subsecuente construcción de infraestructura hidráulica creada por la SRH.

Por otra parte, las dos sequías más importantes registradas en el siglo XX han sido la de 1955 y la del 2000, cuando incluso se llegó a pensar que el lago desaparecería (Filonov et al., 1998). En ambos casos las tierras descubiertas, de propiedad federal, se parcelaron y concesionaron para utilizarse en actividades agropecuarias. Además de que en algunos sitios incluso se llagaron a construir caseríos.

Estas sequías han sido incentivadas por tres factores: la sobreextracción de agua, los cambios en los niveles de precipitación pluvial y la evaporación. Paré (1989) sostiene que a partir de 1940 las extracciones de agua empiezan a superar a las aportaciones. Desde entonces el lago de Chapala ha sufrido extracción excesiva, debido a que desde 1957 Chapala abastece aproximadamente el $60 \%$ de la demanda de agua de Guadalajara, la segunda ciudad más poblada del país, con un incremento poblacional de 2814029 en 1990 a 4434878 en el 2010. Aunado a esto existe un importante descenso en el aporte de agua recibida en el lago proveniente del río Lerma, lo cual se debe a que el agua se queda almacenada en las 552 presas distribuidas a lo largo de la cuenca para abastecer sistemas de irrigación (Tereshchenko et al., 2002).

En lo referente al turismo en el lago de Chapala, este se inició desde la época de Porfirio Díaz, en 1895. Según Ojeda (2007), la élite de esa época comienza una relación diferente con el lago. Esa nueva población establece cotos privados en la ribera del lago y rompe así el uso colectivo de los espacios públicos. Además de que desde sus inicios el turismo en la zona chapálica se desarrolló para las élites de las ciudades de Guadalajara y la Ciudad de México, así como turistas o jubilados migrantes provenientes de Estados Unidos y Europa. Sin embargo, no fue sino hasta principios de los cincuenta que se desarrolló, por parte del gobierno del estado, el malecón y parte de la infraestructura actual para atraer el turismo. Las figuras 4 y 5 , fotografías de los años treinta y cuarenta, muestran cómo en la ribera del lago, en la parte noroccidental, se empiezan a sustituir las zonas dedicadas a la pesca y las lanchas de pescadores por infraestructura turística y lanchas para recorridos turísticos. 

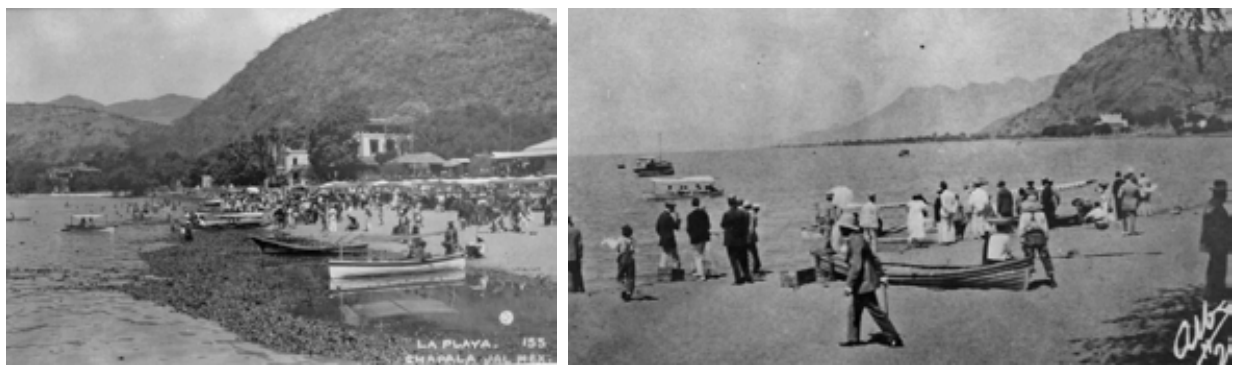

Figuras 4 y 5. Ribera del lago de Chapala a principios del siglo XX

Fuente: Archivo histórico del municipio de Chapala

\section{La actualidad de la ribera del lago de Chapala}

En la actualidad cada uno de los municipios que bordean la ribera del lago ha redefinido sus actividades económicas y esto continúa determinando el uso del suelo (figura 6). La actividad pesquera, aunque con dificultades, se sigue llevando a cabo en todos los municipios, pero es más importante para los municipios de Venustiano Carranza, en Michoacán, y Jamay y Chapala en Jalisco. La agricultura y la ganadería también se llevan a cabo en todos los municipios, aunque algunos sobresalen por su productividad. En la figura 6 se puede observar cómo la agricultura y el pastizal son actividades predominantes en el uso del suelo de la ribera chapálica.

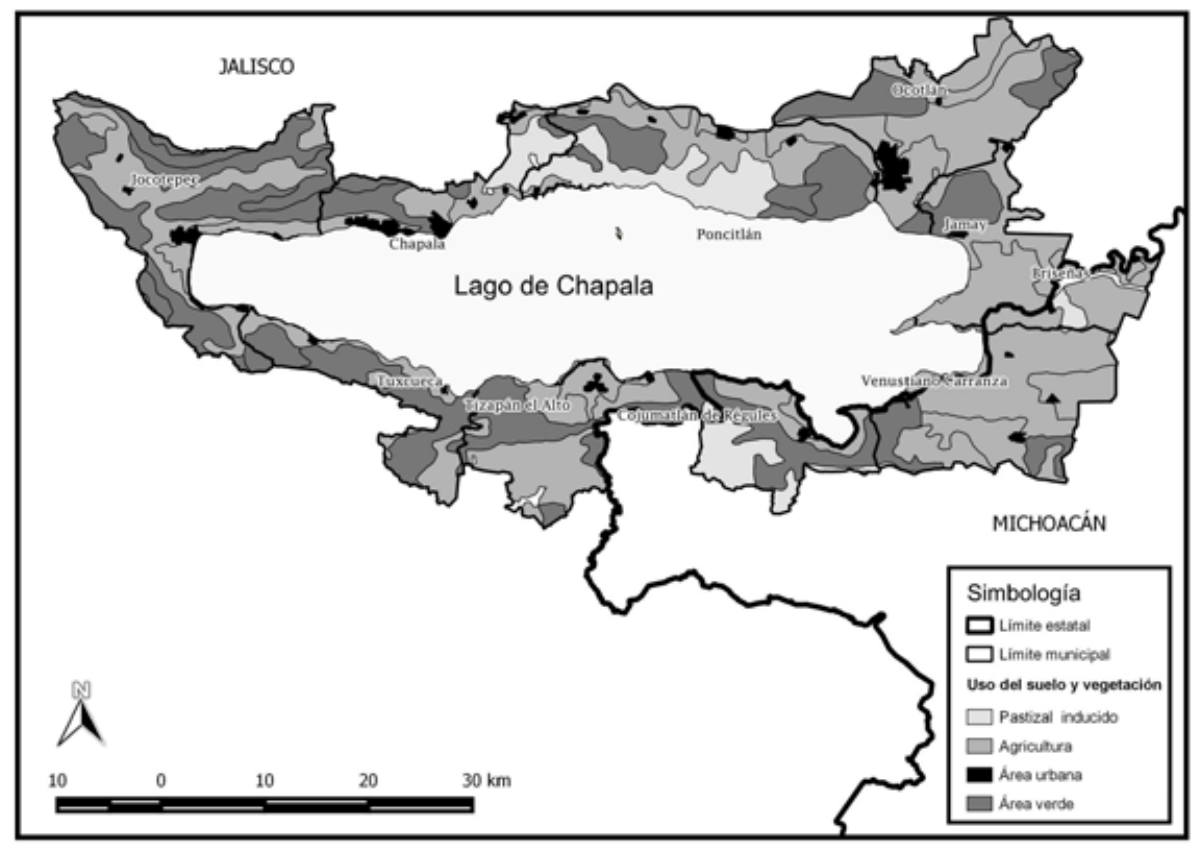

Figura 6. Uso del suelo en la ribera del lago de Chapala

Fuente: INEGI (2000). Edición: María Guadalupe Sámano Leyva 
La infraestructura turística en la ribera del lago predomina en la parte noroccidental y corresponde al estado de Jalisco, principalmente en las localidades de Chapala, Ajijic, San Antonio, San Juan Cosalá y Jocotepec. Una de las formas en que esto se evidencia es el número de hoteles registrados en los municipios que bordean el lago por la Secretaría de Turismo (Sectur) del estado de Jalisco, ya que en 1999 había 55 y actualmente hay 163. Por otro lado, en los poblados de Jamay, la Palma y Petatán, estos dos últimos en Michoacán (municipios de Venustiano Carranza y Cojumatlán, respectivamente), se han llegado a construir desde caseríos hasta restaurantes y malecones, incluso en la zona federal, lo cual es ilegal (véase figuras 7, 8, 9 y 10).

Uno de los casos más radicales es la ciudad de Chapala, la cual se describía como "un pequeño, hermoso y pacífico pueblo de pescadores" (Alba, 1954, véase figura 3). Sin embargo, de un "pueblito de pescadores" ha pasado a convertirse en un destino turístico de fama internacional. En los poblados de Jalisco el turismo está caracterizado por la inmigración de ciudadanos estadounidenses y canadienses, principalmente. A finales del siglo XX la zona turística conformada por Chapala, Ajijic y Poncitlán recibía, aproximadamente, 300000 turistas, de los cuales se calcula que $21 \%$ eran extranjeros (Pare, 1989). En el 2015 Sectur-Jalisco reportó 1148157 turistas, con un 16\% de extranjeros.

Existen municipios donde el turismo es una de las principales actividades económicas y la mayor parte de las casas ribereńas al lago pertenecen a extranjeros jubilados que han migrado a la zona. En municipios como Chapala y Ajijic, en el estado de Jalisco, es difícil encontrar zonas de desembarque pesquero, o incluso pescadores, ya que la infraestructura portuaria fue construida para las lanchas que pasean a los turistas, no para los pescadores (figura 7).

La infraestructura construida para el turismo se puede ver en la figura 7, que es una fotografía del puerto de Chapala. En las figuras 8, 9 y 10 se muestra cómo los espacios para la pesca son cada vez más reducidos, debido a que se van ocupando con infraestructura urbana que incluye viviendas, malecones y restaurantes. La figura 9 muestra la construcción de un restaurante que invade de manera desordenada e ilegal el litoral costero del lago, en tanto que en la figura 10 se puede ver el reducido espacio que hay para que las lanchas de pescadores puedan salir a pescar.

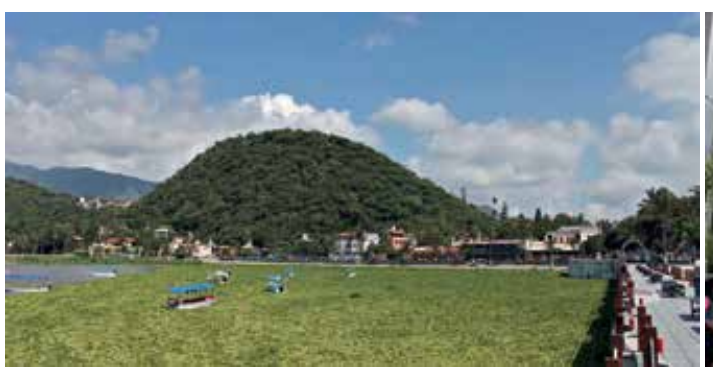

Figura 7. Costa actual de Chapala

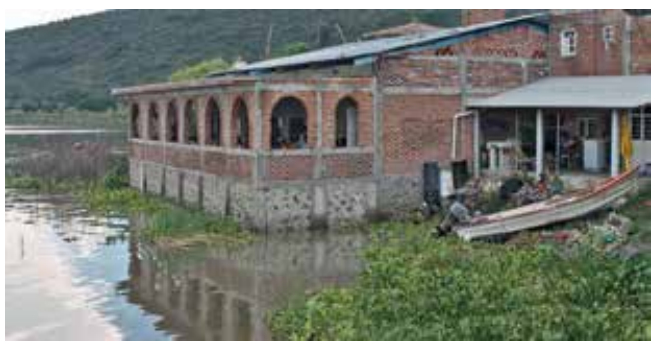

Figura 9. Construcción ilegal en Petatán

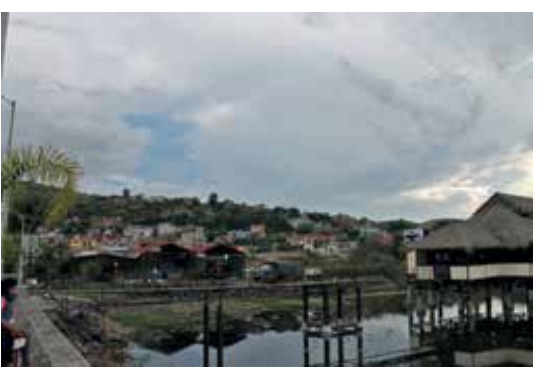

Figura 8. Infraestructura urbana en La Palma

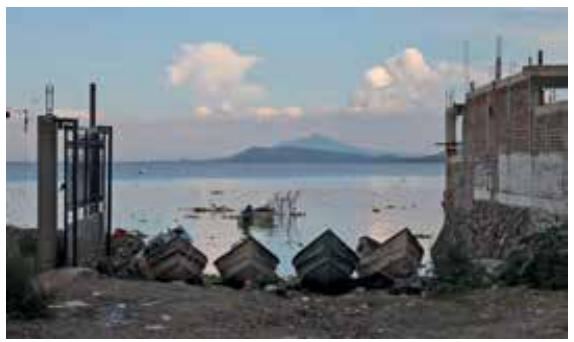

Figura 10. Últimos espacios para la pesca

Fuente: fotografías tomadas por los autores 
El incremento del turismo va aunado a un crecimiento poblacional de los municipios que bordean el lago y su consecuente expansión urbana. Como se puede observar en la tabla 1, en la generalidad de dichos municipios se ha dado un incremento poblacional. Esto sin duda implica una mayor presión para los recursos costeros y los proporcionados por el lago, además de que la zona se sigue considerando eminentemente agrícola y el estado de Michoacán es el primer productor agrícola del país. En los municipios donde el crecimiento es negativo, ello se debe a la migración ante la escasez de recursos y oportunidades de trabajo para los habitantes.

Tabla 1. Dinámica poblacional: municipios en la ribera del lago de Chapala

\begin{tabular}{lcccc}
\hline \multicolumn{1}{c}{ Municipio } & $\begin{array}{c}\text { Tasa de creci- } \\
\text { miento decenal } \\
1970-1980\end{array}$ & $\begin{array}{c}\text { Tasa de creci- } \\
\text { miento decenal } \\
1980-1990\end{array}$ & $\begin{array}{c}\text { Tasa de creci- } \\
\text { miento decenal } \\
1990-2000\end{array}$ & $\begin{array}{c}\text { Tasa de creci- } \\
\text { miento decenal } \\
\text { 2000-2010 }\end{array}$ \\
\hline Chapala, Jal. & 25.94 & 72.53 & -17.79 & 12.42 \\
\hline Cojumatlán, Mich. & 13.59 & -3.80 & -4.87 & 0.76 \\
\hline Jamay, Jal. & 32.30 & 13.63 & 10.51 & 8.15 \\
\hline Jocotepec, Jal. & 10.52 & 24.99 & 15.47 & 18.06 \\
\hline La Barca, Jal. & 14.81 & 13.24 & 11.81 & 8.77 \\
\hline Ocotlán, Jal. & 38.20 & 17.65 & 20.90 & 10.41 \\
\hline Poncitlán, Jal. & 21.92 & 19.90 & 26.56 & 18.57 \\
\hline Tizapán el Alto, Jal. & 19.25 & 11.79 & 0.86 & 5.52 \\
\hline Tuxcueca, Jal. & 2.60 & -1.00 & 10.11 & 3.39 \\
\hline Venustiano Carranza, Mich. & -4.51 & 28.73 & -2.45 & 4.20 \\
\hline
\end{tabular}

Fuente: censos de población y vivienda de 1970 a 2010

En lo que respecta a la pesca, en la primera mitad del siglo XX esta era la segunda fuente de trabajo de los habitantes de las comunidades ribereñas del lago (Ojeda, 2007) y hasta hace algunos años la captura proveniente de sus aguas representaba entre el 50 y el $60 \%$ del volumen pesquero del estado de Jalisco. Sin embargo, la apropiación de los espacios costeros, los cambios en el nivel de agua junto con la contaminación e introducción de especies exóticas, carpa y tilapia, han afectado las especies nativas y los volúmenes de captura. Además de que entre el 2010 y el 2011 se hicieron declaraciones en medios sobre los niveles de contaminación del lago que provocaron una disminución del consumo de pescado con la consecuente caída de los precios. La situación del lago y el conjunto de estos factores conforman, sin duda, un grupo de amenazas para la continuidad de la pesca artesanal lacustre.

Todo esto tiene impactos negativos en la actividad pesquera y estos se reflejan en los volúmenes de captura. Estos últimos, como puede apreciarse en la figura 11, tienen una tendencia a la baja, y en el 2000 coinciden con el periodo de sequía severa que sufrió el lago. En general, la figura 11 muestra cómo los volúmenes de captura coinciden con las oscilaciones en el nivel de agua del lago.

Como puede apreciarse, la apropiación del lago y su territorio costero, ya sea por construcción de infraestructura, por contaminación o por turismo, cada día va reduciendo más los espacios que originalmente ocupaban los pescadores. 


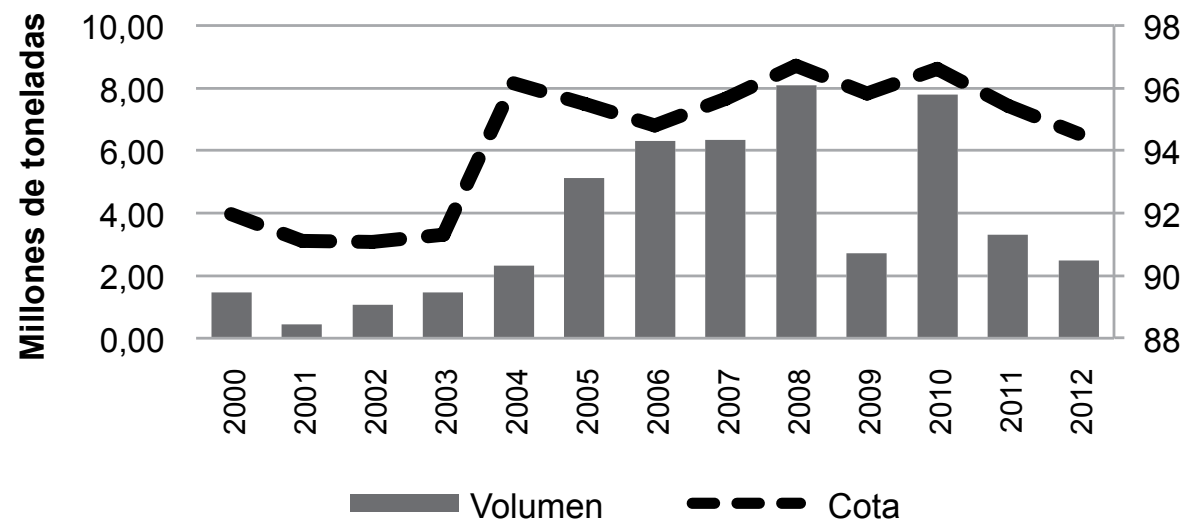

Figura 11. Volumen de captura y niveles de agua en el lago de Chapala

Fuente: elaboración propia con datos de la Secretaría de Agricultura, Ganadería, Desarrollo Rural, Pesca y Alimentación (Sagarpa, 2013)

\section{Conclusiones}

Este trabajo presenta la evolución histórica de los procesos que han transformado el lago de Chapala. Esto se refiere, en parte, a las características naturales del lago; sin embargo, los impactos negativos se acentúan con el cambio climático y las actividades antropogénicas que resultan en procesos de eutrofización, contaminación, introducción de especies exóticas, construcción de infraestructura y cambios de uso del suelo en su zona costera. Esto provoca la disminución de su superficie, afecta sus niveles de agua y capacidad productiva e incluso ha deteriorado su belleza paisajística en una parte de su ribera. Lo anterior ha tenido una relación directa con la visión gubernamental de satisfacer las demandas de los sectores productivos (Cotler, 2004), pero con una visión productivista que reclama una expansión de la frontera agropecuaria y una sobreextracción de agua, sin considerar el impacto en otras actividades productivas como la pesca ni, sobre todo, los daños a los ecosistemas lacustres y las economías locales.

Desafortunadamente, la historia de deterioro y amenazas al lago de Chapala no es un caso exclusivo; se trata de factores comúnmente identificados en diferentes cuencas hídricas en diversas regiones del mundo.

Existen ejemplos de diferentes cuerpos de agua continentales en otras regiones cuyas funciones originales han sido transformadas y su productividad ha disminuido. En la mayoría de ellos se desarrollaba una importante actividad pesquera como son los casos de los grandes lagos en la frontera de Canadá y Estados Unidos, el mar de Aral, o bien los grandes lagos africanos (Beeton, 2002). Sin embargo, al igual que el lago de Chapala, factores antropogénicos como los ya mencionados han resultado en el deterioro de una importante actividad de pesca comercial.

De la misma manera que las pesquerías del lago de Chapala, los grandes lagos de Canadá pasaron por un periodo en el que se advertía sobre su pesca contaminada y también han sido afectados por la disminución de la precipitación pluvial y el cambio climático.

Las transformaciones y el deterioro sufrido por otros cuerpos de agua continentales también tienen que ver con cambios en los mercados y modelos de desarrollo. Como ejemplo de ello podemos citar el caso del mar de Aral, el cual ha sido víctima de un deterioro aceptado a cambio de favorecer el desarrollo económico regional (Golubev, 1996). 
Así como en el lago de Chapala, las comunidades costeras a los lagos africanos son altamente dependientes de los recursos que obtienen de estos, tanto para su alimentación como para su subsistencia. Sin embargo, la deforestación, la erosión del suelo y la desertificación son factores que están degradando a estos lagos (Hecky y Bugenyi, 1992). Al mismo tiempo, la sobrepesca y la introducción de especies exóticas han afectado los volúmenes de captura y las comunidades biológicas se han visto alteradas (Turner, 1994). Todos estos factores coinciden con la región chapálica.

Por otra parte, las desecaciones sufridas en la ciénaga de Chapala también son el reflejo de un abrupto cambio de uso del suelo inducido por la mano del hombre, con impactos en los sistemas de mercado locales y una redefinición del paisaje lacustre y de las economías locales. Después de que la actividad pesquera ocupara ejércitos de hombres y mujeres, y fuera la base de la gastronomía local (Ortiz, 2001), la casi desaparición de las especies nativas y la disminución en los volúmenes de captura han redefinido: el mercado laboral —incentivando la migración—, los mercados locales e incluso los platillos tradicionales que utilizaban estas especies. Se ha inducido una transformación sociocultural y ambiental.

El actual proceso de deterioro en la cuenca Lerma-Chapala, relacionado con la intensa actividad industrial, las actividades agropecuarias y el incremento poblacional, que a su vez son responsables de la sobreexplotación del agua, de los cambios de uso del suelo y del azolvamiento del lago, también afecta otros cuerpos de agua importantes en México como el lago de Cuitzeo, el segundo cuerpo de agua continental más importante del país, que además de ubicarse en la misma cuenca comparte un proceso histórico de degradación con el lago de Chapala (Mendoza et al., 2007).

Para ambos embalses políticas públicas implementadas desde un centralismo burocrático han dejado de lado la consulta y la inclusión de los actores sociales locales, como son los distintos grupos de pescadores. En ambos lagos se ha presentado la situación de una afectación a la fauna acuática nativa por la introducción de especies exóticas. Y en ambos se han implementado políticas públicas que priorizan la extracción de agua para uso pecuario y agrícola, en tanto que en el ámbito de la actividad pesquera se establecen vedas y la prohibición de uso de algunas artes de pesca no del todo efectivas (Franco et al., 2011).

Las razones que dan cabida a estos acontecimientos las encontramos en el enfoque que ha predominado en la planeación del desarrollo nacional. Este ha sido un enfoque dirigido por los intereses político-económicos de quienes detentan poderes fácticos, con una visión de corto plazo, lo cual da lugar a un deterioro ambiental cuyo costo social no ha sido considerado. El caso del lago de Chapala se convierte, de esta manera, en una muestra de los resultados de políticas poco articuladas y mal implementadas que han causado graves deterioros en los recursos naturales de México.

\section{Agradecimientos}

Los autores agradecen el apoyo del Programa de Apoyo a Proyectos de Investigación e Innovación Tecnológica (PAPIIT IA300215) de la Universidad Nacional Autónoma de México y del proyecto SIP-20150431 de la Secretaría de Investigación y Posgrado del Instituto Politécnico Nacional. Asimismo, se reconoce la importancia de los comentarios y las observaciones hechas por los revisores y el comité editorial para enriquecer el presente manuscrito.

\section{Referencias}

Alba, A. D. (1954). Los enemigos del lago. Recuperado de http://chapala. mex. tl/frameset. php

Beeton, A. M. (2002). Large freshwater lakes: present state, trends, and future. Environmental Conservation, 29(1), 21-38. 
Covarrubias, F. y Ojeda, A. (2009). Poblamiento y agricultura en la Ciénaga de Chapala. Oaxaca: Instituto de Investigaciones Sociales y Humanas, SC-IPN.

Cardona Carlin, N. (2004). Definición del área de influencia y análisis de la dinámica socioeconómica de la cuenca Lerma-Chapala. Gaceta Ecológica, (71).

Clavijero, F. J. (1964). Descripción de la ciudad de Los Ángeles o Angelópolis. Tesoros Documentales de México. Siglo XVIII, 324-328.

Comisión Nacional del Agua (Conagua, 2011). Estadísticas del agua en México. México: Secretaría de Medio Ambiente y Recursos Naturales.

Coops, H., Beklioglu, M. y Crisman, T. (2003). The role of water-level fluctuations in shallow lake ecosystemsworkshop conclusions. Hydrobiologia, 503-509(1-3), 23-27.

Cotler, H. (2004). La cuenca Lerma-Chapala: algunas ideas para un antiguo problema. Gaceta Ecológica, (71).

Cotler, H. y Gutiérrez, S. (2005). Inventario y evaluación de presas de la cuenca Lerma-Chapala. México: INE.

Dávila Moreno, M. E. N. (2014). El surgimiento de la ganadería en la ciénega de Chapala (Michoacán, México). El caso de la hacienda Guaracha (siglos XVI-XIX). Revista de Historia Regionaly Local, 6(11),185-218.

Dudgeon, D. (2000). Large-scale hydrological changes in tropical Asia: prospects for riverine biodiversity. BioScience, 50(9), 793-806.

Filonov, A. E., Tereshchenko, I. E. y Monzón, C. O. (1998). Oscillations of the hydrometeorological characteristics in the region of Lake Chapala for intervals of days to decades. Geofisica Internacional, 37(4), 293-307.

Franco, C., Galicia, L., Durand, L. y Cram, S. (2011). Análisis del impacto de las políticas ambientales en el lago de Cuitzeo (1940-2010). Investigaciones Geográficas, (75), 7-22.

García de León, A. (1988). Las grandes tendencias de la producción agraria. En E. Semo (Coord.), Historia de la cuestión agraria mexicana. 1. El siglo de la hacienda 1800-1900 (pp. 13-86). México: Siglo XXI, Centro de Estudios Históricos del Agrarismo en México.

Gattenlöhner, U. (2006). Lake tourism - Examples from the worldwide Living Lakes Network. Recuperado de http://www.globalnature.org/

Golubev, G. N. (1996). Caspian and Aral seas: two different paths of environmental degradation. VerhandlungenInternationale Vereinigung für theoretische und angewandte Limnologie, 26, 159-166.

González Santana, O. M. (2009). El manejo de los recursos hidricos y el control del territorio. El caso del valle de Ecuandureo en el occidente de México. Ponencia presentada en el XII Encuentro de Geógrafos de América Latina, Montevideo, Uruguay.

Hecky, R. E. y Bugenyi, F. W. B. (1992). Hydrology and chemistry of the African Great Lakes and water quality issues: problems and solutions. Mitteilung Internationale Vereinigung fuer Limnologie, (23), 45-54.

Kolding, J. y van Zwieten, P. A. M. (2012). Relative lake level fluctuations and their influence on productivity and resilience in tropical lakes and reservoirs. Fisheries Research, (115-116), 99-109.

Mendoza, M. E., Bocco, G., López Granados, E. y Bravo, M. (2007). Tendencias recientes de las superficies ocupadas por el lago de Cuitzeo: un enfoque basado en percepción remota, sistemas de información geográfica y análisis estadístico. Investigaciones Geográficas, (64), 43-62.

Moncayo-Estrada, R., Lyons, J., Escalera-Gallardo, C. y Lind, O. (2012). Long-term change in the biotic integrity of a shallow tropical lake: A decadal analysis of the Lake Chapala fish community. Lake and Reservoir Management, (28), 92-104. 
Moreno García, H. (1989). Haciendas de tierra y agua. México: El Colegio de Michoacán.

Ojeda Sampson, A. (2007). Contaminación y uso del agua. En F. Cobarruvias Villa (Coord.), El potencial turístico del tramo Michoacán de la ribera del lago de Chapala (pp. 33-38). México: Instituto de Investigaciones Sociales y Humanas.

Ojeda Sampson, A., Covarrubias Villa, F. y Arceo Ortega, M. G. (2008). El proceso de antropización del lago de Chapala, secuencia. Revista de Historia y Ciencias Sociales, (71), 103-129.

Ortiz Segura, C. (2001). Todo tiempo pasado fue mejor, o la pesca en el lago de Chapala antes de la desecación de su ciénaga. Gazeta de Antropología, (17).

Paré, L. (1989). Los pescadores de Chapala y la defensa de su lago. Tlaquepaque, México: Instituto Tecnológico y de Estudios Superiores de Occidente (Iteso).

Sandoval, F. (1994). Pasado y futuro del lago de Chapala. Guadalajara, México: UNED.

Sarch, M. T. y Birkett, C. (2000). Fishing and farming at Lake Chad: Responses to lake-level fluctuations. The Geographical Journal, 166(2), 156-172.

Simonian, L. (1995). Defending the land of the jaguar. A history of conservation in México. Austin: University of Texas Press.

Tereshchenko, I., Filonov, A., Gallegos, A., Monzón, C. y Rodríguez, R. (2002). El Niño 1997-98 and the hydrometeorological variability of Chapala, a shallow tropical lake in Mexico. Journal of Hydrology, (264), 133-146.

Turner, G. F. (1994). Fishing and the conservation of the endemic fishes of Lake Malawi. Advances in Limnology Physycs Review, (47), 777-780.

Von Bertrab, E. (2003). Guadalajara's water crisis and the fate of Lake Chapala: a reflection of poor water management in Mexico. Environment and Urbanization, 15(2), 127-140.

Welcomme, R. L., Cowx, I. G., Coates, D., Béné, C., Funge-Smith, S., Halls, A. y Lorenzen, K. (2010). Inland capture fisheries. Philosophical Transansactions, (365), 2881-2896.

Wolfe, M. (2013). The historical dynamics of Mexico's groundwater crisis in La Laguna: Knowledge, resources and profit, 1930s-1960s. Mexican Studies, 29(1), 3-35. 